\title{
Clinical observations of using LiuWei DiHuang bolus combined with acarbose to treat early diabetic nephropathy
}

\author{
Chao Chen MSc${ }^{1}$, Chunfang Guo BS², Hong Zheng PhD
}

C Chen, C Guo, H Zheng. Clinical observation of using LiuWei DiHuang bolus combined with acarbose to treat early diabetic nephropathy. Curr Res Integr Med 2015;1(2):20-22.

OBJECTIVE: To observe the clinical effect of LiuWei DiHuang bolus combined with acarbose to treat early diabetic nephropathy.

METHOD: A total of 117 diabetic outpatients who met the study inclusion criteria were randomly assigned to an observation group and a control group. The control group was treated with glucoba, while the observation group was treated with LiuWei DiHuang bolus combined with acarbose.
RESULTS: The observation group exhibited an impressive cure rate $(91.5 \%)$, which was better than the total effective rate $(\mathrm{P}<0.05)$.

CONCLUSION: Administration of LiuWei DiHuang bolus combined with acarbose appeared to be highly effective in treating early diabetic nephropathy, and may be a clinically valuable treatment alternative.

Key Words: Clinical effect; Diabetic nephropathy; Early stage; LiuWei DiHuang bolus; Type II diabetes
$\mathrm{D}$ iabetes mellitus (DM) is a common and frequently encountered disease, the incidence of which is increasing steadily alongside improvement in living standards, the acceleration of population aging and changing lifestyles. It is reported that there are 114 million patients with DM in China, the world's most populous country, of whom $>90 \%$ are diagnosed with type II DM, the highest number of DM patients. The medications prescribed for type II DM by Western physicians are typically more effective in controlling blood glucose levels compared with complications. Complications of DM remain the leading cause of death in diabetic patients. Diabetic nephropathy (DN), also known as diabetic glomerulosclerosis, is the major lesion of diabetic microangiopathy, for which the complication rate is $20 \%$ to $40 \%$; primary symptoms are chiefly characterized by proteinuria, edema, hypertension and, later, renal insufficiency or even kidney failure. In the view of traditional Chinese medicine (TCM), diabetes, mainly caused by disorders of diet, emotion and daily routine, is a type of 'diabetes', whose basic pathogenesis stems from deficiency of both qi and yin. Qi deficiency fails to drive the yin liquid to flow, which causes yin liquid to accumulate in dampness to produce phlegm, and more phlegm generates more fire in the day. In turn, fire will be harmful to yin liquid flow, which leads to qi deficiency. More serious would be that fire colonizing the upper-Jiao causeing upper diabetes, middleJiao for middle diabetes and lower-Jiao for lower diabetes. According to TCM, which views the kidney as water viscera and the radix of qi, the key question in treating DM is how to nourish the kidney and yin, and to benefit qi for promoting the production of fluid. Acarbose, an $\alpha$-glycosidase inhibitor commonly used for clinical type II DM, can slow the intestinal absorption of glucose, which results in reducing blood glucose levels, especially after meals. In our hospital, a common method of treating patients with early DN caused by deficiency of qi and yin involves a LiuWei DiHuang pill in combination with acarbose, which has shown promising clinical effects.

\section{METHODS}

A total of 117 subjects ( 67 men, 50 women) were chosen from a group of patients diagnosed with type II DM and early DN between January and December 2013 in the authors' hospital. The mean ( \pm SD) age was $42.1 \pm 8.3$ years (range 37 to 64 years), and the range of duration of DM was one to nine years (mean $4.2 \pm 1.1$ years). All patients were randomly assigned into one of two groups: a control group $(n=58)$ and a treatment group $(n=59)$. The control group consisted of 32 men and 26 women, ranging in age from 37 to 64 years (mean $42.1 \pm 8.3$ years), with a duration of DM ranging from one to nine years (mean $4.2 \pm 1.1$ years); the control group included 35 men and 24 women, ranging in age from 37 to 64 years (mean $42.1 \pm 8.3$ years), with a duration of DM ranging from one to nine years (mean $4.2 \pm 1.1$ years). The differences in sex, age and course of disease were not significant $(P>0.05)$, which makes the comparison valid.

\section{Inclusion criteria}

1. Meeting the WHO diagnostic criteria for the diagnosis of type II DM;

2. In line with the TCM differentiation on diabetes of deficiency of both qi and yin, with syndromes of soreness and weakness in the waist and knees, dispiritedness and lassitude, lack of qi and no desire to speak, lusterless complexion, emaciation, loose stool, pale and dingy tongue or ecchymosis, or thready and uneven heart rate;

3. Patients who previously took drugs to treat blood glucose without improvement: glycated hemoglobin ( HbAlc) $>6.5 \%$, fasting blood glucose $(\mathrm{FBG})>7.0 \mathrm{mmol} / \mathrm{L}$;

4. Traces of microprotein in urine ( $30 \mathrm{mg} / \mathrm{L}$ to $180 \mathrm{mg} / \mathrm{L})$;

5. Undergoing diet or exercise therapy;

6. Never taken acarbose in the past;

7. Body mass index $<35 \mathrm{~kg} / \mathrm{m}^{2}$;

${ }^{1}$ Department of Traditional Chinese Medicine; ${ }^{2}$ Outpatient Department, the First Affiliated Hospital of Jinan University, Guangzhou, China; ${ }^{3}$ School of Fundamental Medical Science, Guangzhou University of Chinese Medicine, Guangzhou, China

Correspondence: Mr Chao Chen, Department of Traditional Chinese Medicine, the First Affiliated Hospital of Jinan University,

613 Huangpu Avenue W, Tianhe District, Guangzhou, Guandong, 510630, China. Telephone 13392692159, e-mail tcchen@189.cn 
TABLE 1

Comparison of blood glucose levels and urine trace protein indexes of the two groups before and after treatment

\begin{tabular}{lcccc}
\hline & \multicolumn{2}{c}{ Control group $(\mathbf{n}=58)$} & \multicolumn{2}{c}{ Observation group (n=59) } \\
\cline { 2 - 5 } Index & $\begin{array}{c}\text { Before } \\
\text { treatment }\end{array}$ & $\begin{array}{c}\text { After } \\
\text { treatment }\end{array}$ & $\begin{array}{c}\text { Before } \\
\text { treatment }\end{array}$ & $\begin{array}{c}\text { After } \\
\text { treatment }\end{array}$ \\
\hline FBG, mmol/L & $9.85 \pm 1.25$ & $8.53 \pm 1.42$ & $9.92 \pm 1.25$ & $7.21 \pm 1.33^{*}$ \\
PBG, mmol/L & $13.54 \pm 2.73$ & $11.32 \pm 2.89$ & $14.07 \pm 2.32$ & $9.25 \pm 1.08^{*}$ \\
HbA1c, \% & $11.02 \pm 2.34$ & $10.32 \pm 2.53$ & $11.31 \pm 1.97$ & $8.79 \pm 1.17^{\star}$ \\
Microalbuminuria, & $81.24 \pm 11.93$ & $76.42 \pm 12.33^{*}$ & $82.31 \pm 10.64$ & $45.22 \pm 13.17^{*}$
\end{tabular}
$\mathrm{mg} / \mathrm{L}$

${ }^{*}$ Compared with the result before treatment $(P<0.05)$. FBG Fasting blood glucose; HBA1c Glycated hemoglobin; PBG Postprandial blood glucose

8. Patients who signed informed consent to agree to participate in the present study and cooperate with therapy according to the research programmer.

Exclusion criteria

1. Patients with type $1 \mathrm{DM}$;

2. Pregnant women with gestational DM;

3. Patients with liver and kidney failure, severe infection, diabetic ketoacidosis or thyroid disorders;

4. Allergies to drugs used in the present study;

5. Individuals with severe gastrointestinal disease.

\section{Therapy}

Subjects in the control group took acarbose (Bayer Healthcare Ltd, Beijing) orally before meals, according to the following directions: first three days $50 \mathrm{mg}$ per dose, from fourth day $100 \mathrm{mg}$ per dose. Subjects in the comparison group took acarbose the same as those in control group as well as LiuWei DiHuang (concentrated pill form, produced by Beijing Tongrentang Technology Development Co, Ltd, China), according to the following directions: eight pills per dose, three times per day. Clincal efficacy was evaluated after three months.

\section{Indications for further observation}

FBG and postprandial glucose testing was performed once every two weeks in both groups; testing for glycated hemoglobin (HbAlc) was performed once per month; testing for microalbuminuria, body mass index, total cholesterol, triglyceride, high-density lipoprotein and lowdensity lipoprotein occurred before and after therapy. Adverse reactions during the treatment were recorded.

\section{Response evaluation criteria}

1. Significant effect: Clinical symptoms disappear or are significantly improved; signs of life are returning to normal or significantly improved; the value of FBG measurements is $<7.2 \mathrm{mmol} / \mathrm{L}$ and the blood glucose level $2 \mathrm{~h}$ after a meal $<8.3 \mathrm{mmol} / \mathrm{L}$; and the level of microalbuminuria decreases $>75 \%$ compared with before treatment;

2. Valid effect: Either clinical symptoms or abnormal vital signs of subjects significantly improved; the value of FBG measurements is $<10.0 \mathrm{mmol} / \mathrm{L}$ and the PBG level $2 \mathrm{~h}$ after a meal is $<8.3 \mathrm{mmol} / \mathrm{L}$; and the level of microalbuminuria decreases $>50 \%$ than before treatment;

3. Invalid effect: Neither clinical symptoms nor abnormal vital signs of patients shows significant improvement or worsened; outcomes from standard laboratory tests have failed to meet the definitions of effective treatment.

Statistical analysis

SPSS version 13.0 (IBM Corporation, USA) was used to analyze the data. Data are presented as mean \pm standard deviation, and differences
TABLE 2

Comparison of body mass index (BMI) and blood lipid indexes of the two groups before and after treatment

\begin{tabular}{lcccc}
\hline & \multicolumn{2}{c}{ Control group $(\mathbf{n = 5 8})$} & Observation group $(\mathbf{n = 5 9})$ \\
\cline { 2 - 5 } $\begin{array}{c}\text { Index } \\
\text { treatment }\end{array}$ & $\begin{array}{c}\text { After } \\
\text { treatment }\end{array}$ & $\begin{array}{c}\text { Before } \\
\text { treatment }\end{array}$ & $\begin{array}{c}\text { After } \\
\text { treatment }\end{array}$ \\
\hline $\begin{array}{c}\text { Cholesterol total, } \\
\mathrm{mmol} / \mathrm{L}\end{array}$ & $28.32 \pm 3.89$ & $27.96 \pm 3.39$ & $28.90 \pm 3.21$ & $21.46 \pm 3.40^{*}{ }^{\dagger}$ \\
$\begin{array}{l}\text { Glycerin trilaurate, } \\
\mathrm{mmol} / \mathrm{L}\end{array}$ & $2.24 \pm 0.43$ & $2.27 \pm 0.39$ & $2.32 \pm 0.40$ & $1.67 \pm 0.36^{\star \dagger}$ \\
$\mathrm{HDL}, \mathrm{mmol} / \mathrm{L}$ & $1.25 \pm 0.31$ & $1.29 \pm 0.26$ & $1.22 \pm 0.34$ & $1.79 \pm 0.23^{\star \dagger}$ \\
$\mathrm{LDL}, \mathrm{mmol} / \mathrm{L}$ & $3.03 \pm 0.26$ & $2.89 \pm 0.30$ & $3.05 \pm 0.35$ & $2.56 \pm 0.42^{\star \dagger}$ \\
$\mathrm{BMI}$ & $28.64 \pm 5.86$ & $28.45 \pm 6.77$ & $28.93 \pm 5.84$ & $23.55 \pm 3.21^{\star \dagger}$ \\
\hline
\end{tabular}

${ }^{*} P<0.05$ Compared with the result before treatment; ${ }^{\dagger} P<0.05$ compared with control group. HDL High-density lipoprotein; LDL Low-density lipoprotein

TABLE 3

Comparison of clinical efficacy in the two groups

\begin{tabular}{lccccc}
\hline Group & Cases & $\begin{array}{c}\text { Remarkable } \\
\text { result }\end{array}$ & Effective & Invalid & $\begin{array}{c}\text { Total effective } \\
\text { rate, \% }\end{array}$ \\
\hline Control group & 58 & 28 & 17 & 13 & 77.6 \\
$\begin{array}{l}\text { Observation } \\
\text { group }\end{array}$ & 59 & 35 & 19 & 5 & $91.5^{\star}$ \\
\hline
\end{tabular}

Data presented as $n$ unless otherwise indicated. ${ }^{*} P<0.05$ compared with control group

between the two groups were assessed using independent-sample $t$ tests. Differences were considered to be statistically significant at $\mathrm{P}<0.07$.

\section{RESULTS}

Comparison of laboratory indicators between two groups

Laboratory indicators of the two groups before and after treatment are shown in Tables 1 and 2. The results indicate that subjects in the two groups improved with regard to blood glucose levels, urine protein, blood lipid indexes and body mass index after treatment. The laboratory indexes of the treatment group significantly improved, and there was a statistically significant difference compared with those before treatment (ie, $\mathrm{P}<0.05$ ). After treatment, the laboratory indexes of the observation group were also more improved than the control group $(\mathrm{P}<0.05)$.

Comparison of clinical efficacy between the two groups

The comparison shown in the Table 3. The observation with definite cure rate of $91.5 \%$ is better than the comparison with definite cure rate of $77.6 \%$. The comparison difference of cure rate in the two groups is true statistically with $\mathrm{P}<0.05$.

\section{Adverse reactions in the two groups}

Adverse reactions in the two groups are characterized by low blood sugar, flatulence and diarrhea. There was one case of hypoglycemia, two cases of flatulence and one case of diarrhea in the treatment group, and one case of hypoglycemia and one case of flatulence in the control group. There were no significant differences in adverse reactions between the two groups.

\section{DISCUSSION}

In recent years, the incidence of diabetes around the world has been increasing consistently, and China is set to become the world's most populous nation with regard to patients with DM. High blood glucose levels are not fatal, but many tissues and organs can be damaged, which leads to various complications including fundus disease, cardiac dysfunction, renal failure, and cerebrovascular diseases due to longterm exposure of a high-glucose environment in the body; these can seriously affect the patients' quality of life, and may even be life-threatening. 
Therefore, controlling blood glucose level is an effective way to reduce the incidence of diabetes and prevent chronic complications. Diabetes is an illness caused by metabolic disorders often accompanied by high cholesterol levels, hypertension and other diseases, which, in turn, will aggravate the symptoms of diabetes and accelerate the development of complications. For these reasons, it is very important to curb high cholesterol and high blood pressure when controlling blood sugar. DN is a microvascular complication caused by diabetes with early symptoms, which are not easy to detect, being unconsciously ignored by the patient. As DN progresses, it will gradually develops into renal failure and persist without early intervention. The uncertainty remains for pathogenesis of $\mathrm{DN}$ at present in medicine.

TCM considers diabetes as one category of 'diabetes', primarily yin deficiency and dry heat; patients first often feel thirsty, then drink more and diabetes more, resulting in emaciation. The illness causes permanent damage to collaterals and kidneys, and caused blood stasis. If this occurs in the arteries and veins of kidneys, blood stasis will block blood circulation; if it continues for a longer time, impairments will cause anemia and impair qi. It is a pathological model of qi deficiency and blood stasis because the qi deficiency blocks blood flow and generates more blood stasis. Qi deficiency stops semen assembly to such an extent that edema appears; in addition, the body will lose the ability to maintain warmth, leading the patient to sense malaise and cold. Internal stagnation of the blood induces back aches and blood stagnation in the tongue base. Qi deficiency is associated with uncontrol of liquid and, thus, loss of microscopic protein. The special spirit of TCM in curing diabetes is nourishing kidney and yin, and benefiting qi for promoting production of fluid. LiuWei DiHuang bolus is a vintage prescription, which was initially documented in Specific Formula of Pediatrics (third volume), written by Qian Yi, a medic born in the Song Dynasty. The name given by Qian Yi is Dihuang bolus, which derives from the Kidney bolus, prescribed by Zhang Zhongjing, honored as the Great Master, in his Synopsis Of Prescriptions Of The Golden Chamber. The formula of Qian Yi, whose ingredients are dogwood, dried yams, rhizomaalismatis, cortex moutan and poria, reduces two ingredients from Zhang Zhongjing's and replace the dried rehmannia root for the fried rehmanniae. The element of use a large

\section{REFERENCES}

1. Mao Y, Wei L. Advances in Diabetes Mangement. Shandong Med J 2014;54:99-101.

2. Lu H, Zhang D, Cui N. The progress of surgical treatment of type II diabetes. Chin J Surg Integr Traditional Western Med 2013;19:4802.

3. Wang J. Internal medicine [M]. Beijing: People's Medical Publishing House, 2005:98.

4. Ming L, Ding C, Fang F. Advances in the treatment of diabetes in TCM. Information on Traditional Chinese Medicine 2012;29:112-5.

5. Xie X. The clinical treatment of LiuWei Dihuang pill in diabetes. Journal of North Pharmacy 2014;11:25. quantity dried rehmannia root for its function of nourishing kidneys and yin can replenish semen and nourish marrow, supplied to nobles in ancient China; the element of dogwood can nourish the liver and kidneys and unsmoothen semen, for the reason that kidneys and liver are in the same source according to TCM. The elements of yam can tonify the spleen and yin, and also secure the kidneys. The three elements mentioned make 'three tonics'. With the help of rhizomaalismatis having the effect of removing dampness by dieresis to drain turbidity of kidneys, and of poria being slightly permeable into the spleen to keep it damp and strong when yam involves, and of cortex moutan clearing asthenic fever and neutralizing the dryness of dogwood, the three elements have been called 'three laxatives'. After fully studying the formula, we find that the efficacy of the LiuWei DiHuang bolus is tonifying while draining; that the tonifying determines the draining; that three elements can play a role in tonifying with the others of draining but more tonifying than draining, and that the liver, the kidney and the spleen all are yin-organs, could be nourished, the first two take much more advantages of it. This medicine can cure patients with not only primary but secondary symptoms of early DN and DM. Modern pharmacological research shows that the LiuWei DiHuang bolus has many efficacies, such as improving glucose tolerance, reducing blood glucose levels, improving urine protein, improving diabetes and complications, antioxidation, improving insulin resistance and inhibiting the apoptosis of islet cells. This therapy combines the LiuWei DiHuang bolus with acarbose to treat patients with DM and early DN, which reveals that the two drugs combined in treatment could significantly improve blood glucose, urine microprotein, serum lipids and body mass index; therefore, we can infer from the actual process that combined LiuWei DiHuang bolus and acarbose not only can control blood glucose levels but also relieve the complications of DM to some degree, especially the symptoms of early DN. This makes the combination clinically valuable, and may become popular in the future.

DISCLOSURES: The authors have no financial disclosures or conflicts of interest to declare.

6. Huang Q. Clinical observation of 55 cases of type II diabetes with combined metformin acarbose. Chin J Ethnomed Ethnopharm 2014:23:60-1.

7. Zhu W. Research progress of vitamin D and diabetes and its complications. Guide of China Medicine 2014;12:58-9.

8. Xie Q. The role and research of LiuWei Dihuang pill in the treatment of type II diabetes. Clin J Chin Med 2013;5:116-8.

9. Dai F, Zhang J. Clinical observation of metformin combined with Liuwei Dihuang pill in the treatment of type II diabetes. Practical J Cardiac Cerebral Vasc Dis 2012;20:1887.

10. Li B, Jiang H, Bian R, et al. Progress of the clinical application of Liuwei Dihuang pill. J Pharm Res 2014,33:170-1. 